\title{
Estudio de la influencia del Cu y Ni en la cinética de transformación martensítica inducida por deformación en fundiciones nodulares austemperadas $^{(\bullet)}$
}

\author{
D. Guzmán*, L. Navea*, J. Garín**, C. Aguilar*** y A. Guzmán**
}

Resumen

El objetivo de este trabajo fue estudiar el efecto del cobre y níquel en la cinética de la transformación martensítica inducida por deformación en fundiciones nodulares austemperadas. Las fundiciones utilizadas se fabricaron mediante austemperado, a partir de dos fundiciones nodulares, con diferentes contenidos de cobre y níquel. La deformación se realizó en un laminador de rodillo. La cuantificación de las fases se realizó mediante difracción de rayos X, mientras que la caracterización microestructural se efectuó utilizando microscopía óptica y electrónica de barrido. Se comprobó que la cinética de transformación martensítica inducida por deformación en fundiciones nodulares austemperadas puede ser modelada mediante los modelos de Olson-Cohen y Chang et al. Basándose en los resultados obtenidos de estos ajustes, se concluye que tanto el níquel como el cobre dificultan la transformación martensítica debido a que estos elementos aumentan la energía de falla de apilamiento de la austenita, además de aumentar su estabilidad termodinámica.

Palabras clave Fundiciones nodulares austemperadas; Cinética de transformación martensítica inducida por deformación; Difracción de rayos X.

\section{Study of the influence of $\mathrm{Cu}$ and $\mathrm{Ni}$ on the kinetics of strain-induced martensite in austempered ductile cast iron}

\begin{abstract}
The objective of this work was to study the influence of copper and nickel on the kinetics of strain-induced martensite in austempered ductile cast iron. The austempered ductile cast irons were obtained from two ductile cast irons with different copper and nickel contents by means of austempering treatment. The deformation was carried out using a rolling mill. The quantification of the phases was obtained by means of $\mathrm{X}$ ray diffraction, while the microstructural characterization was carried out using optical and scanning electron microscopy. It was proved that the kinetics of strain-induced martensite in austempered ductile cast iron can be modeled using the equations proposed by OlsonCohen and Chang et al. Based on the results obtained from these analyses, it is possible to conclude that the nickel and copper complicate the martensite transformation because these elements increase the staking fault energy of the austenite and its thermodynamic stability.
\end{abstract}

Keywords

Austempered ductile cast iron; Kinetics of strain-induced martensite; X-ray diffraction.

\section{INTRODUCCIÓN}

Las fundiciones nodulares austemperadas (Austempered Ductile cast Iron, ADI) han tenido un creciente uso debido a sus propiedades mecánicas de tenacidad (100-140 J) ${ }^{[1]}$, ductilidad (2-15\%), resistencia a la tracción $(1200-1400 \mathrm{MPa})$ y al desgaste $^{[2]}$ combinado con sus menores costos de producción en comparación con los aceros ${ }^{[3]}$.
Las fundiciones nodulares austemperadas ADI, se obtienen a partir de un tratamiento térmico aplicado a las fundiciones nodulares, el cual es conocido como austemperado. Este tratamiento parte con una etapa de austenitizado a temperaturas entre 1093 y $1223 \mathrm{~K}$, el tiempo suficiente para obtener una matriz completamente austenítica, seguido de un tratamiento isotérmico entre 503 y $673 \mathrm{~K}$, por un tiempo que varía habitualmente entre 7,2 y 14,4 ks. Durante este

(•) Trabajo recibido el día 7 de junio de 2012 y aceptado en su forma final el día 3 de abril de 2013.

* Universidad de Atacama, Dpto. de Metalurgia y Centro Regional de Investigación y Desarrollo Sustentable de Atacama (CRIDESAT), Avenida Copayapu 485, Copiapó, Chile. E-mail: danny.guzman@uda.cl.

** Universidad de Santiago de Chile, Dpto. de Ingeniería Metalúrgica, Avenida Bernardo O’Higgins 3363, Santiago, Chile.

*** Universidad Técnica Federico Santa María, Dpto. de Ingeniería Metalúrgica y de Materiales, Avenida España 1680, Valparaíso, Chile. 
tratamiento isotérmico suceden dos reacciones. En la primera de ellas la austenita $(\gamma)$ se descompone en ferrita $(\alpha)$ acicular y austenita de alto carbono $\left(\gamma_{\mathrm{AC}}\right)$, microconstituyente conocido como ausferrita ${ }^{[4]}$.

$$
\gamma \rightarrow \alpha+\gamma_{A C}
$$

Si la fundición es mantenida a esta temperatura durante un tiempo prolongado, se verifica una segunda reacción, donde la austenita de alto carbono se descompone en ferrita y carburo- $\varepsilon$ :

$$
\gamma_{\mathrm{AC}} \rightarrow \alpha+\varepsilon
$$

En este caso, el carburo- $\varepsilon$ torna frágil al material, por lo que esta reacción debe ser evitada.

Se ha establecido que la mejor combinación de propiedades mecánicas se obtiene al término de la primera reacción, es decir, cuando la fundición está compuesta por nódulos de grafito y ausferrita ${ }^{[5]}$. La estabilidad de la austenita en la ausferrita se debe principalmente a su alto contenido de carbono, el cual se encuentra entre 1,6 y 2,0 \% en masa ${ }^{[6]}$ y a la presencia de elementos aleantes, como por ejemplo manganeso, cobre, níquel y molibdeno ${ }^{[7]}$. Estos elementos aleantes, además, aumentan la austemperabilidad de la aleación, evitando la formación de perlita y ferrita durante el enfriamiento hasta la temperatura de austemperado, permitiendo de esta manera, que la formación de ausferrita se complete a lo largo de toda la sección de la pieza ${ }^{[8]}$. La supresión de la aparición de la cementita se logra por la adición de silicio, cuya cantidad habitualmente varía entre 1,5 y $3,0 \%$ en masa ${ }^{[9]}$.

Como se mencionó anteriormente, ya que el níquel y en menor proporción el cobre, retardan la reacción de austemperado y la formación de bainita $^{[10]}$, estos elementos influyen en la obtención de la estructura ausferrítica, la cual afecta las propiedades mecánicas de la fundición. En este sentido, se ha reportado que a medida que aumenta la cantidad de austenita retenida en la ausferrita, se produce un aumento de la tenacidad ${ }^{[11-13]}$, la ductilidad ${ }^{[13}$ y 14$]$ y la resistencia a la fatiga ${ }^{[15}$ y 16$]$, lo cual lleva consigo una disminución en el esfuerzo de fluencia ${ }^{[14}$ y 17$]$ de estas aleaciones.

Bajo deformación plástica, la austenita puede absorber energía y transformarse en martensita (fenómeno TRIP) $)^{[4}$ y 6]. Se ha establecido que esta transformación juega un rol importante en la resistencia al desgaste ${ }^{[18]}$ y la capacidad de endurecimiento por deformación ${ }^{[6]}$ que presentan estas aleaciones, en comparación a otras fundiciones.

La figura 1 presenta la dependencia de la energía libre de la austenita y martensita en función de la temperatura. Se aprecia que a $T_{0}$ ambas fases poseen la misma energía libre $\left(G_{\gamma}=G_{\alpha}\right)$. Para temperaturas menores a $\mathrm{M}_{\mathrm{s}}$ la formación de la martensita es posible, sin embargo entre el intervalo de temperaturas sobre $\mathrm{M}_{\mathrm{s}}$ y bajo $\mathrm{T}_{\mathrm{o}}$, no es posible obtener martensita espontáneamente debido a que la diferencia de energía libre entre ambas fases no alcanza un valor crítico de energía, $\Delta \mathrm{G}_{\text {crit }}$. No obstante, la martensita puede formarse sobre la $\mathrm{M}_{\mathrm{s}}$ siempre y cuando se logre un aumento de la energía libre de la austenita, por ejemplo mediante deformación, así la diferencia de energía entre ambas fases será mayor al valor crítico ${ }^{[19]}$. La máxima temperatura a la cual puede ocurrir la transformación martensítica inducida por deformación se denomina $\mathrm{M}_{\mathrm{d}}$; sobre esta temperatura la diferencia de energía es menor a $\Delta \mathrm{G}_{\text {crit }}$.

Aun cuando la martensita producida en las fundiciones ADI presenta un alto contenido de carbono, el cual le confiere un carácter frágil, las fundiciones nodulares austemperadas, debido a su particular microestructura, pueden presentar una tenacidad y ductilidad comparable a una fundición maleable con una resistencia a la tensión dos veces superior ${ }^{[18]}$. En este sentido, Chatterjee y Bhadeshia ${ }^{[20]}$ estudiaron la relación existente entre la tendencia al agrietamiento en aceros de alto carbono ( $1 \%$ masa $\mathrm{C}$ ) y el tamaño de las placas de martensita. Ellos sugirieron que existe un tamaño crítico de las placas de martensita por debajo del cual no presentan agrietamiento cuando el material es deformado, debido a la dificultad de transferir la carga sobre estas pequeñas placas. De acuerdo con este argumento, las fundiciones nodulares austemperadas que presentan

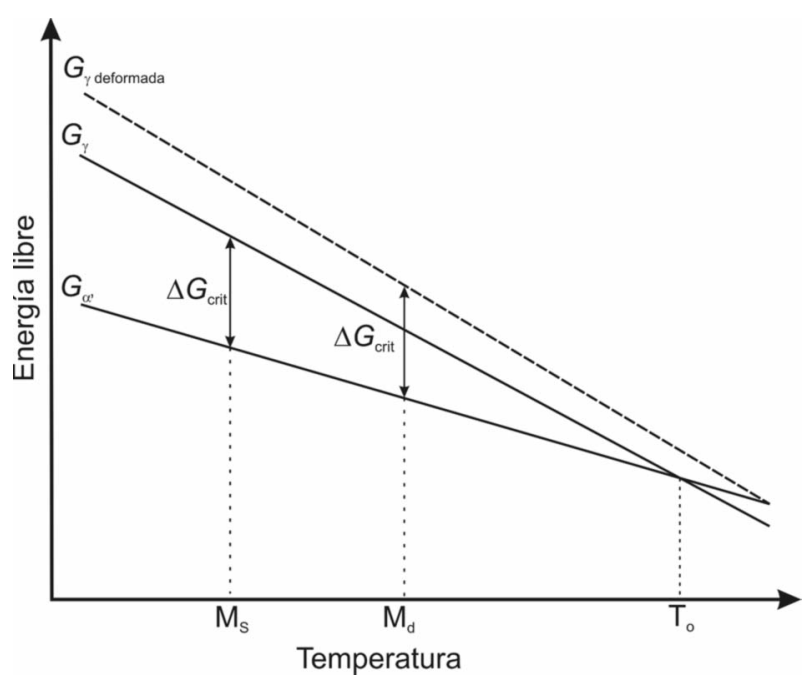

Figura 1. Esquema del diagrama de energía libre de la transformación martensítica inducida por deformación.

Figure 1. A schematic free energy diagram of deformation induced martensitic transformation. 
martensita inducida por deformación, podrían ser visualizadas como un material reforzado con fibras, ya que aun cuando la fibra sea frágil, ésta no fragilizará al material compuesto, siempre y cuando la fibra sea menor que un tamaño crítico.

Existen básicamente dos modelos para relacionar la cantidad de martensita transformada y la deformación plástica aplicada a muestras metálicas. Olson y Cohen ${ }^{[21]}$ propusieron un modelo basado en la efectividad de las intersecciones entre bandas de deslizamiento como sitios preferenciales de nucleación de la martensita. Este modelo relaciona la fracción volumétrica de martensita trasformada, $f^{\alpha^{\prime}}$, en función de la deformación plástica, $\varepsilon$, según la ecuación (3):

$$
f^{\alpha^{\prime}}=1-\exp \left\{-\beta[1-\exp (-\alpha \varepsilon)]^{n}\right\}
$$

donde el parámetro $n$ es un exponente fijo, que para aceros inoxidables austeníticos asume un valor de 4,5 . El parámetro $\alpha$ se encuentra relacionado con la velocidad de formación de las bandas de deslizamiento, la cual depende de la energía de falla de apilamiento y la velocidad de deformación, mientras que el parámetro $\beta$ se encuentra relacionado con la máxima cantidad de martensita transformada.

Por otro lado, Chang et al. ${ }^{[19]}$ propusieron un modelo considerando que la transformación martensítica inducida por deformación plástica puede ser considerada como un continuo proceso de relajación de energía interna acumulada durante el proceso de deformación. La ecuación (4) describe el modelo propuesto por estos investigadores:

$$
\frac{f}{f_{\mathrm{s}}}=1-\exp \left[-\beta\left(\varepsilon-\varepsilon_{0}\right)^{\mathrm{n}}\right]
$$

donde $f$ es la fracción de martensita transformada y $f_{s}$ el máximo valor de martensita transformada por deformación. El parámetro $\varepsilon_{o}$ es la deformación crítica (mínima) para comenzar la transformación martensítica. El parámetro $\beta$ representa la estabilidad de la austenita retenida, que es función de la composición y de la temperatura a la cual se realiza la deformación, mientras que el parámetro $n$, denominado parámetro del modo de deformación, representa la velocidad de formación de los núcleos de martensita durante el proceso de deformación plástica. Este parámetro depende de la composición y del tipo de deformación a la que está sometido el material; para aceros inoxidables austeníticos se ha encontrado que $n$ es igual a 2,2 cuando la deformación se realiza en modo tracción ${ }^{[19]}$.

Aun cuando la reacción martensítica inducida por deformación plástica en fundiciones nodulares austemperadas es importante debido a su efecto en las propiedades mecánicas de estas aleaciones, no existen trabajos en bibliografía que estudien el efecto de los elementos aleantes sobre la cinética de esta transformación. Basado en lo expuesto, el presente trabajo tuvo como objetivo estudiar el efecto del níquel y el cobre sobre la cinética de trasformación martensítica inducida por deformación plástica en fundiciones nodulares austemperadas, mediante los modelos propuestos por Olson-Cohen ${ }^{[21]}$ y Chang et al. ${ }^{[19]}$

\section{PROCEDIMIENTO EXPERIMENTAL}

Las fundiciones nodulares austemperadas utilizadas en este estudio se fabricaron a partir de dos fundiciones nodulares, una sin aleantes y otra aleada con níquel y cobre. Se utilizó un horno de inducción Inductotherm de $30 \mathrm{Kg}$ de capacidad, con crisol de alta alúmina. Las coladas se efectuaron en un molde tipo Y de 25,4 mm de espesor, diseñado según la norma ASTM A 395. La composición química de las fundiciones nodulares, fue obtenida mediante espectrometría de emisión óptica en un equipo Spectrolab. Los resultados obtenidos pueden ser apreciados en la tabla I.

El ciclo de tratamiento térmico se realizó en tres etapas: austenitizado, austemperado y templado en agua. La etapa de austenitizado se realizó en un horno tubular marca Dietert modelo Varitemp, con atmósfera controlada de argon a $1193+/-5 \mathrm{~K}$ durante $4,8 \mathrm{ks}$. La etapa de austemperado se llevó a cabo en un horno de

Tabla I. Composición química de las fundiciones nodulares utilizadas

Table I. Chemical composition of the ductile cast irons

\begin{tabular}{lccccccccc}
\hline & \multicolumn{8}{c}{ Composición (\% en masa) } \\
\cline { 2 - 9 } & $\mathbf{C}$ & $\mathbf{S i}$ & $\mathbf{M n}$ & $\mathbf{P}$ & $\mathbf{S}$ & $\mathbf{M o}$ & $\mathbf{M g}$ & $\mathbf{C u}$ & $\mathbf{N i}$ \\
\hline Sin aleantes & 3,49 & 2,42 & 0,40 & 0,02 & 0,008 & 0,10 & 0,012 & - & - \\
Con aleantes & 3,46 & 2,37 & 0,42 & 0,02 & 0,006 & 0,16 & 0,015 & 1,03 & 0,393 \\
\hline
\end{tabular}


sales marca Hoskins a $683 \mathrm{~K}$ con un tiempo de $450 \mathrm{~s}$ para la fundición sin elementos aleantes y 5,4 ks para la fundición con presencia de níquel y cobre. El término de la transformación isotérmica se realizó mediante un temple en agua agitada a temperatura ambiente.

Las probetas fueron analizadas metalográficamente para verificar su transformación ausferrítica y luego fueron laminadas en frío, empleando un equipo marca Jolliot. Los porcentajes de deformación fueron $2 \%$ por pasada, hasta un $26 \%$ de deformación. Sobre este porcentaje se verificó el agrietamiento de algunas probetas.

Para el análisis microestructural, las probetas se lijaron utilizando parafina sólida para no destruir los nódulos de grafito presentes en la matriz. A conti-

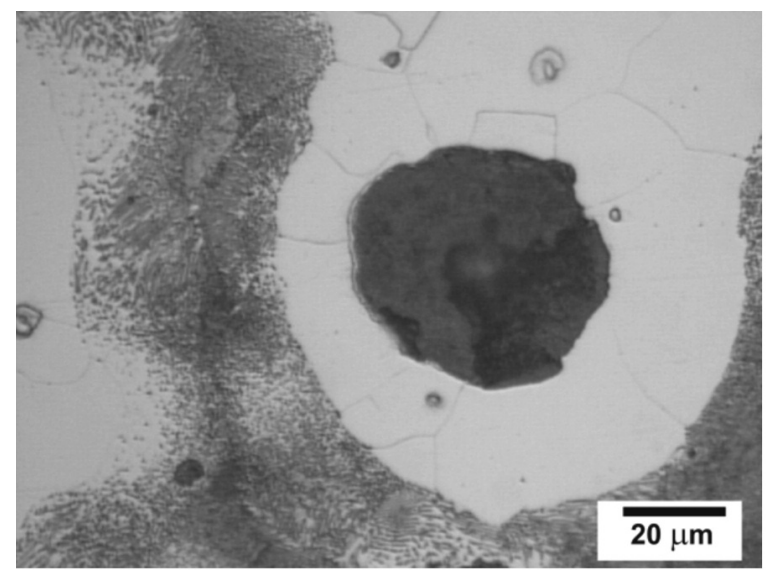

(a) nuación, se pulieron, utilizándose alúmina $1(1 \mu \mathrm{m})$ a $500 \mathrm{rpm}$, alúmina $2(0,1 \mu \mathrm{m})$ a $400 \mathrm{rpm}$ y alúmina 3 $(0,01 \mu \mathrm{m})$ a $300 \mathrm{rpm}$, respectivamente. El reactivo utilizado para revelar la microestructura fue Nital al $2 \%$; el tiempo de ataque varió entre 8 y 12 s. La caracterización metalográfica se realizó en un microscopio óptico marca Zeiss, modelo Axiotech. Además, se realizaron análisis de microscopía electrónica de barrido (MEB) utilizando un equipo Jeol, modelo 5410.

La determinación cuantitativa de fases se realizó mediante difracción de rayos X (DRX). El equipo utilizado fue un difractómetro marca Siemens D500 con radiación característica $\mathrm{K} \alpha \mathrm{Cu}$ y monocromador de grafito. El rango de barrido $2 \theta$ utilizado estuvo

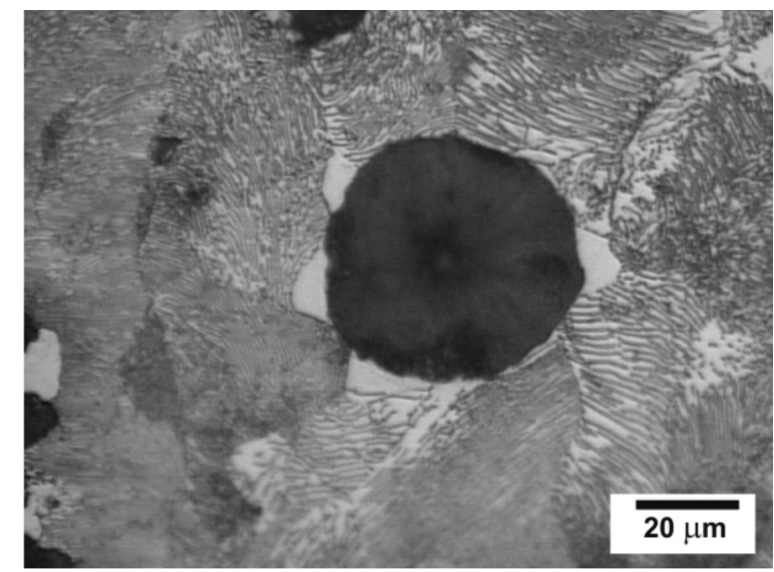

(b)

Figura 2. Micrografías de fundición nodular (a) sin aleantes y (b) aleadas con cobre y níquel.

Figure 2. Micrographs showing ductile cast iron (a) without alloying elements and (b) alloyed with copper and nickel.

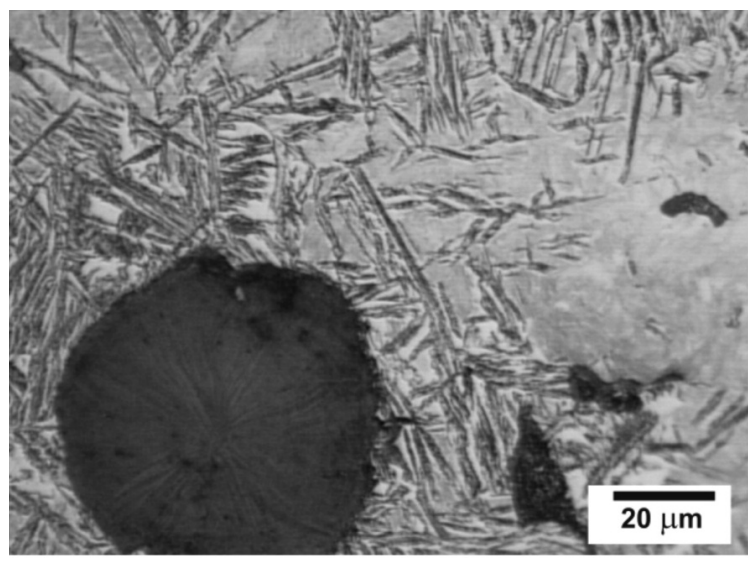

(a)

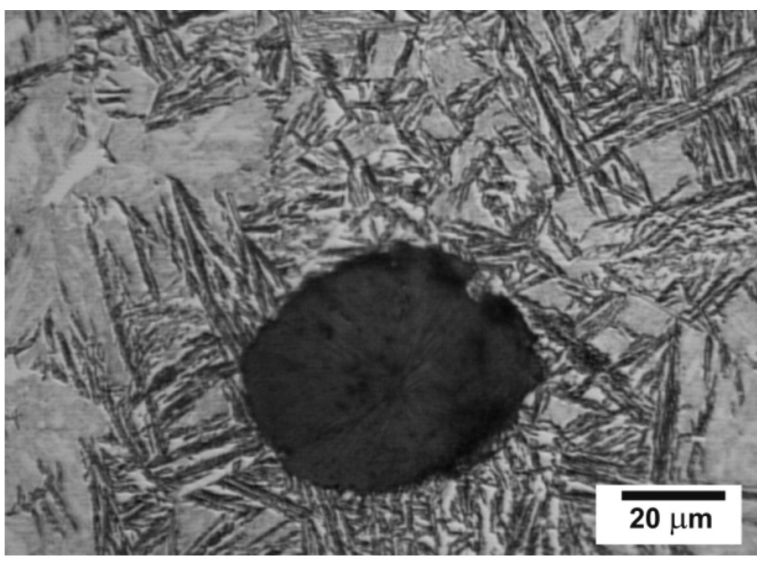

(b)

Figura 3. Micrografías de fundición nodular austemperada (a) sin aleantes y (b) aleadas con cobre y níquel.

Figure 3. Micrographs showing austempered ductile cast iron (a) without alloying elements and (b) alloyed with copper and nickel. 
(a)

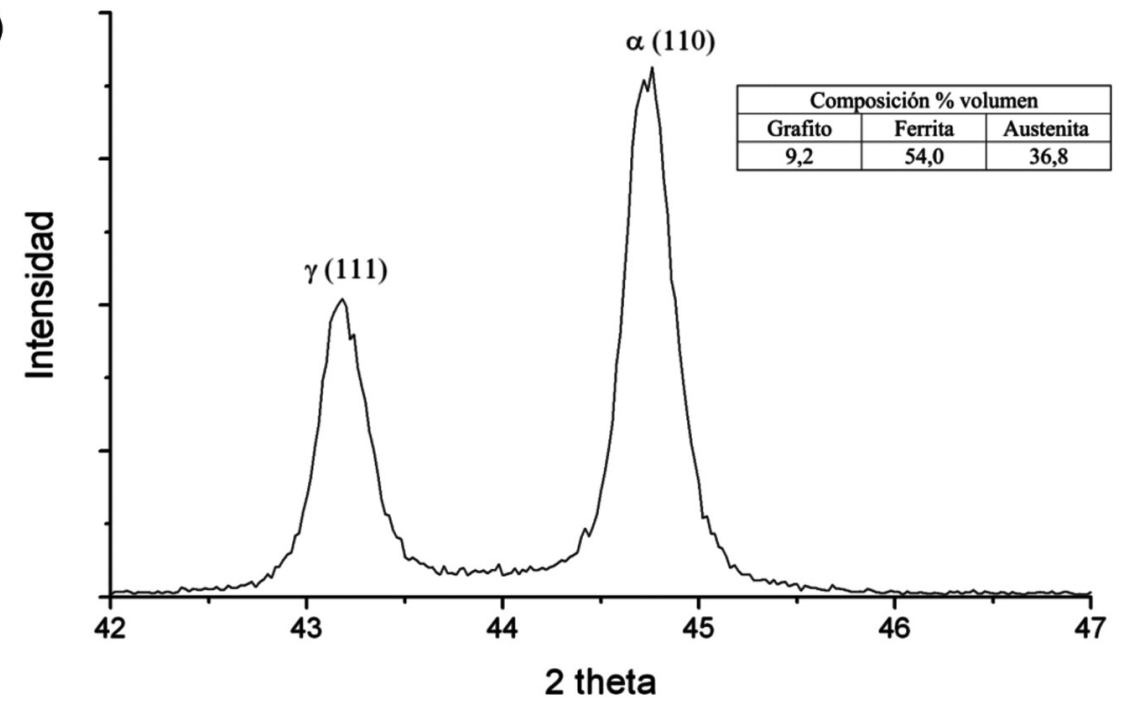

(b)

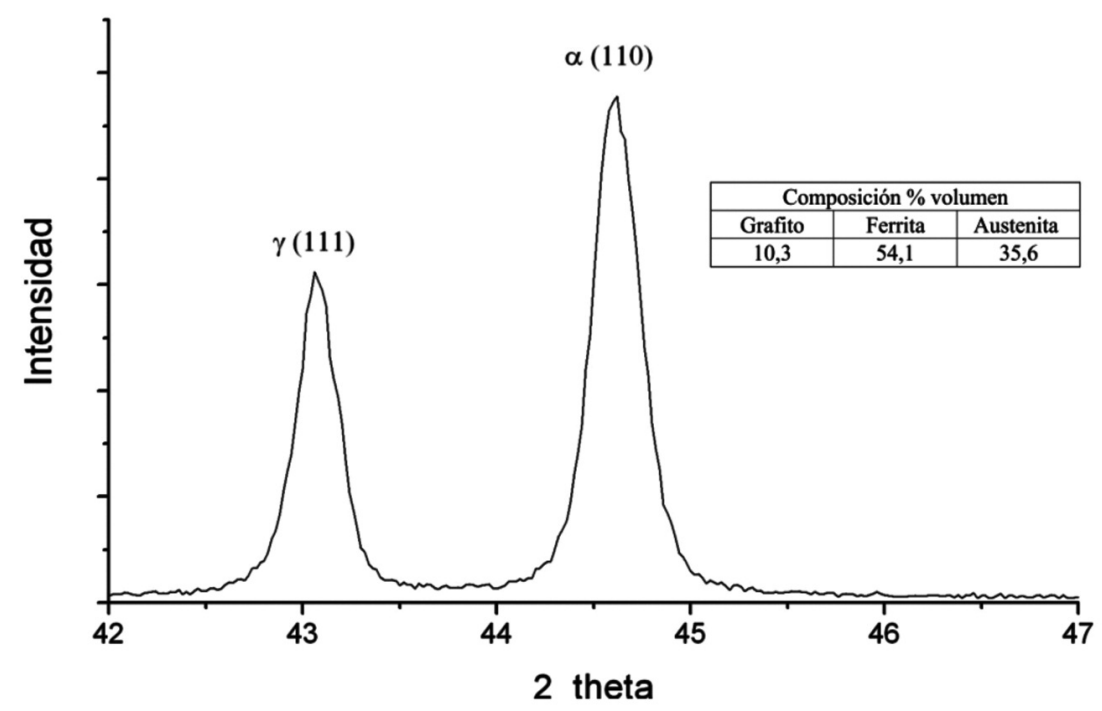

Figura 4. Difractogramas de rayos $X$ de fundiciones nodulares austemperadas (a) sin elementos aleantes y (b) aleadas con cobre y níquel. Se presenta la cuantificación de las fases para cada muestra.

Figure 4. XRD patterns of austempered ductile cast irons (a) without alloying elements and (b) alloyed with copper and nickel. It is shown the phase quantification for each sample. entre 40 y $110^{\circ}$ con un paso de $0,02^{\circ}$ y tiempo de detección de 10 s. Además se utilizaron los algoritmos Profile, Lattice y Lazy -Pulverix.

\section{RESULTADOS Y DISCUSIÓN}

La figura 2 muestra metalografías representativas de las dos fundiciones nodulares bajo estudio. Las fases presentes en cada muestra fueron cuantificadas utilizando quince micrografías para cada caso. En base a los resultados obtenidos se puede establecer que la fundición nodular sin níquel y cobre (Fig. 2(a)) presenta una microestructura llamada "ojo de buey"[22], en la cual los nódulos de grafito (9,2 \% volumen) están rodeados por ferrita $(68,0 \%$ volumen) y perlita fina ( $22,8 \%$ volumen). Por otro lado, la fundición nodular con níquel y cobre (Fig. 2(b)), presenta una menor cantidad de ferrita $(9,4 \%$ volumen) y mayor cantidad de perlita (81,1 \% volumen) que la fundición sin aleantes, lo que puede ser atribuido al efecto perlitizante del cobre ${ }^{[23}$ y 24] y en menor medida del níquel ${ }^{[24]}$. 
(a)

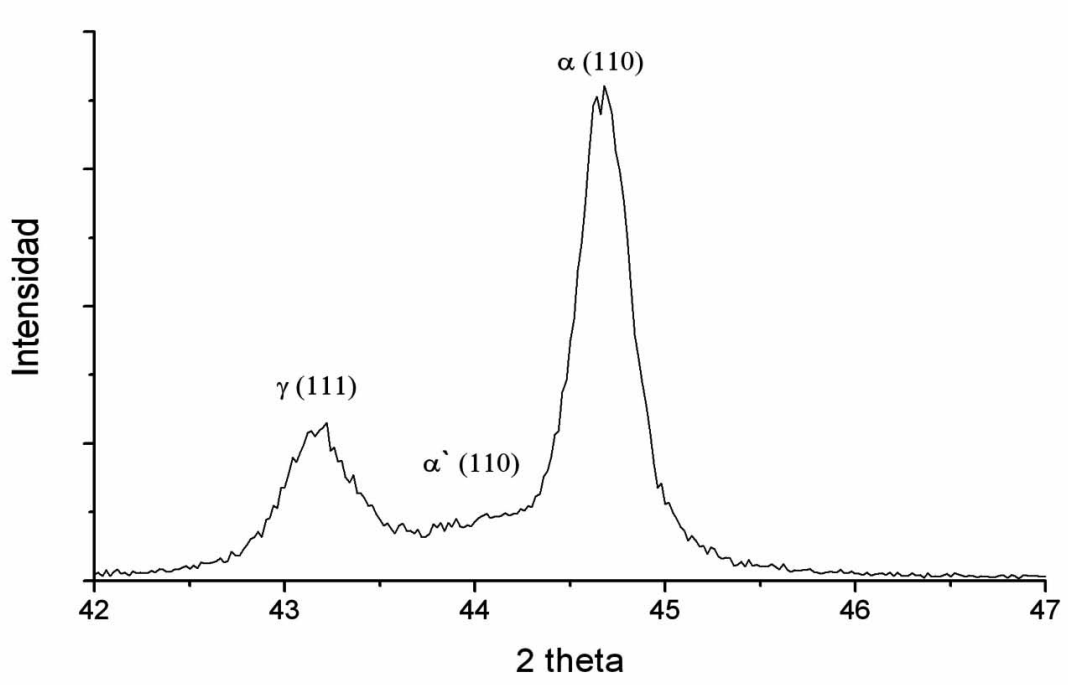

(b)

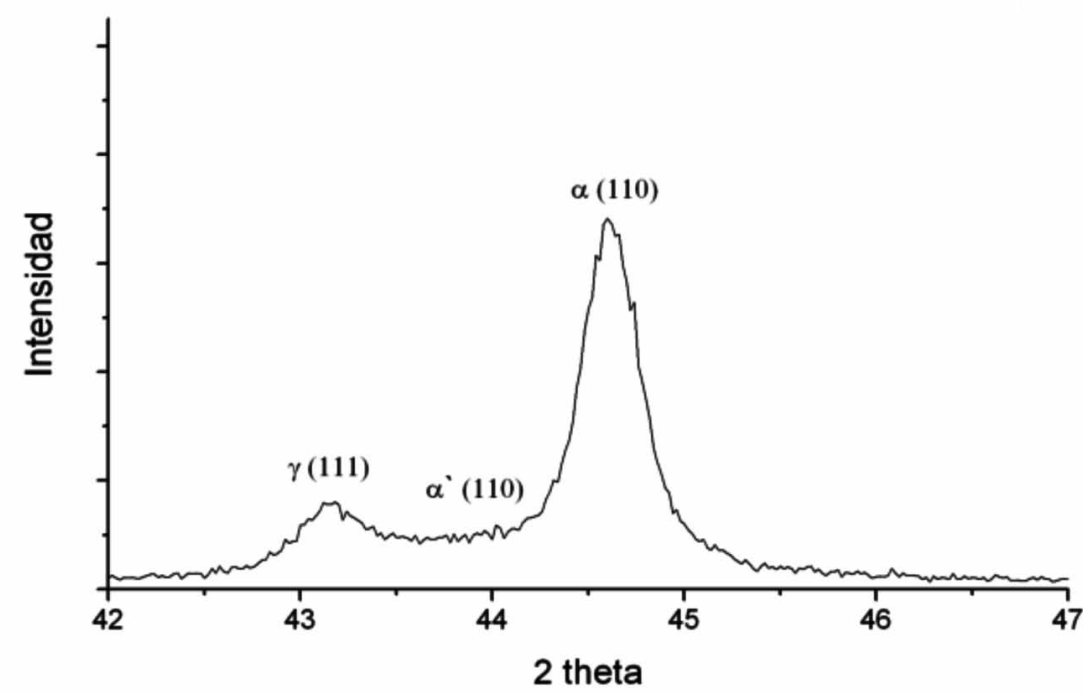

Figura 5. Difractogramas de rayos $X$ de fundiciones nodulares austemperadas con $26 \%$ de deformación (a) sin elementos aleantes y (b) aleadas con cobre y níquel.

Figure 5. XRD patterns of austempered ductile cast irons deformed $26 \%$ (a) without alloying elements and (b) alloyed with copper and nickel.

La figura 3 presenta metalografías representativas de las muestras bajo estudio después de ser sometidas al proceso de austemperado. La cuantificación de las fases se realizó mediante DRX (Fig. 4). Basándose en los resultados obtenidos, se establece que ambas muestras presentan una distribución de ferrita acicular fina en una matriz de austenita (ausferrita), lo que confirma que las condiciones de tratamiento térmico resultaron adecuadas para la fabricación de fundiciones nodulares austemperadas. El hecho de que ambas muestras presenten similares porcentajes de fases, se debe a la diferencia de tiempo durante el tratamiento de austemperado.
La figura 5 presenta los difractogramas de rayos $\mathrm{X}$ de ambas muestras después del proceso de laminación. Si se compara la figura 5 con la figura 4 , se puede observar que las intensidades de los planos (111) de la austenita decrecen con la deformación en frío y se evidencia la aparición de la difracción del plano (110) de la martensita. Estos resultados se corroboran analizando la micrografía MEB obtenida de una muestra laminada un $26 \%$ (Fig. 6). En esta micrografía se aprecia la presencia de agujas de martensita en la matriz austenítica, además de grietas producto de la concentración de esfuerzo debido al trabajo en frío. 
La figura 7 muestra la variación en volumen de martensita inducida por deformación en función del porcentaje de reducción de área para las dos muestras en estudio. La cuantificación de las fases restantes se presenta en la tabla II. Como se observa, el contenido de martensita de las muestras se incrementa desde 0 a un $20 \%$ para la muestra sin alean-

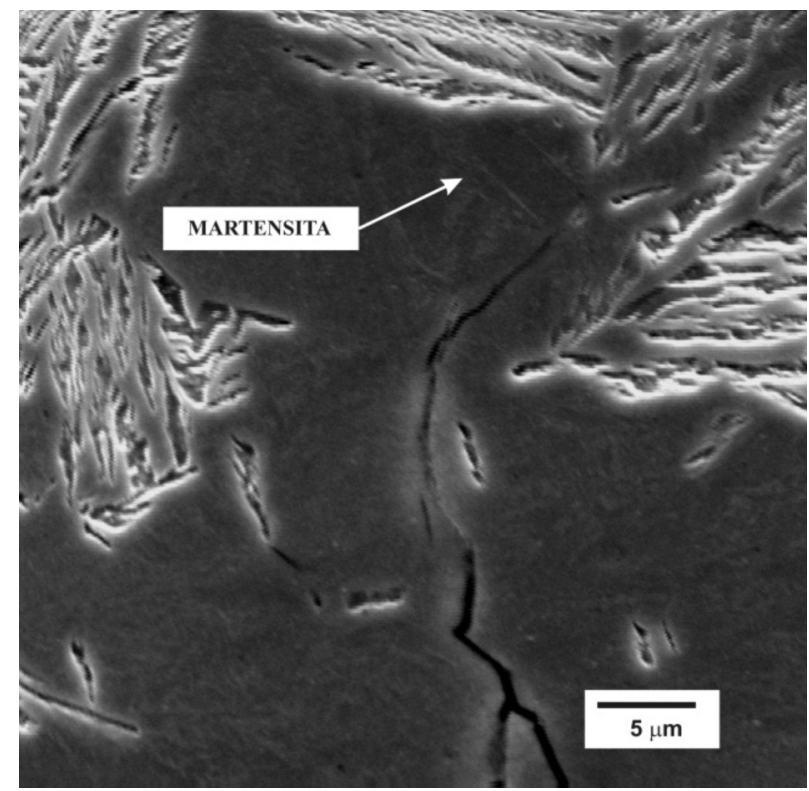

Figura 6. Micrografía de MEB mostrando fundición nodular austemperada sin elementos aleantes deformada un $26 \%$.

Figure 6. SEM micrograph showing austempered ductile cast iron without alloying elements deformed $26 \%$.

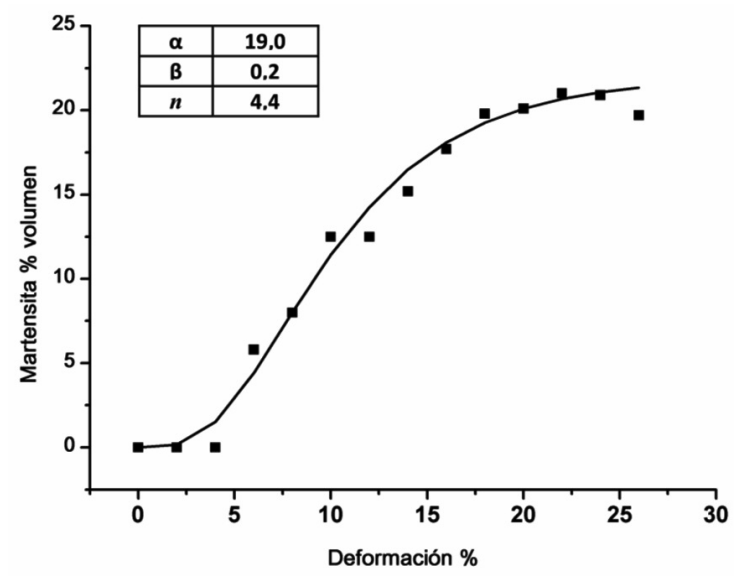

tes y desde 0 a un $24 \%$ para la muestra aleada con cobre y níquel cuando se aplica un $26 \%$ de deformación. Sobre este porcentaje de deformación, las grietas avanzan y el material se fractura.

La figura 8 muestra los ajustes obtenidos utilizando el modelo de Olson y Cohen ${ }^{[21]}$ para ambas muestras. Se determinó que el modelo ensayado

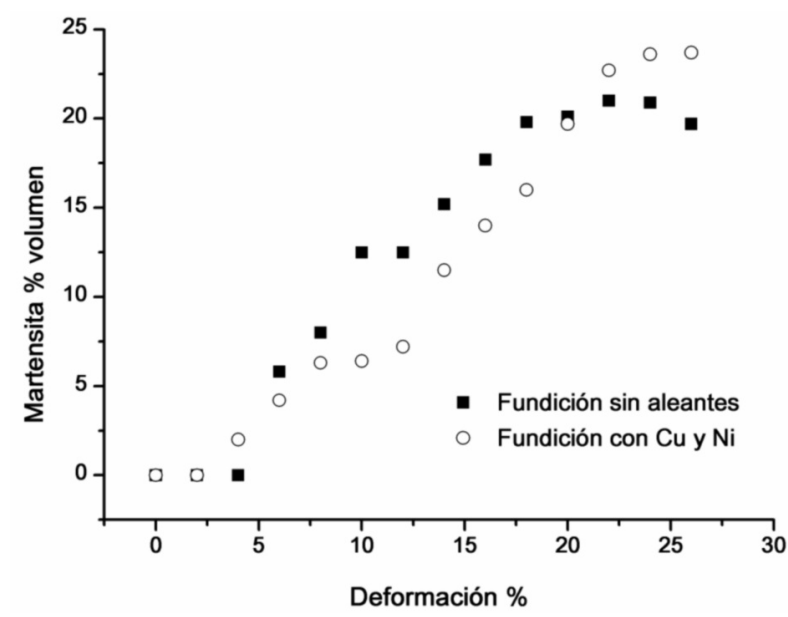

Figura 7. Variación del porcentaje en volumen de martensita inducida por deformación en función del porcentaje de deformación.

Figure 7. Volume fraction of transformed martensite as a function of the strain.

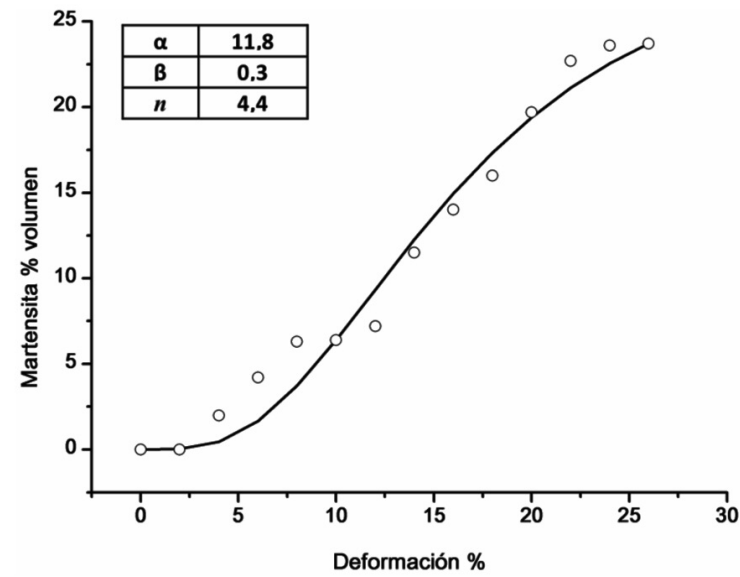

Figura 8. Comparación entre las curvas de transformación calculadas usando el modelo de OlsonCohen y los datos experimentales (a) muestra sin aleantes y (b) muestra aleada con cobre y níquel.

Figure 8. Comparison between the calculated transformation curves obtained using the OlsonCohen model and the experimental data (a) sample without alloying elements and (b) sample alloyed with copper and nickel. 
Tabla II. Cuantificación de fases durante el proceso de laminación

Table II. Quantification of the phases during the rolling process

\begin{tabular}{c|cccc|cccc}
\hline \multirow{2}{*}{$\begin{array}{c}* \\
\text { defor- } \\
\text { mación }\end{array}$} & \multicolumn{6}{c}{ Cuantificación de fases (\% volumen) } \\
\cline { 2 - 8 } & \multicolumn{2}{|c|}{ Fundición sin aleantes Cu y Ni } & \multicolumn{2}{c}{ Fundición aleada con Cu y Ni } \\
\cline { 2 - 8 } & Grafito & Ferrita & Austenita & Martensita & Grafito & Ferrita & Austenita & Martensita \\
\hline \multirow{2}{*}{0} & 9,2 & 54,0 & 36,8 & 0,0 & 10,3 & 54,1 & 35,6 & 0,0 \\
2 & 9,2 & 54,8 & 36,0 & 0,0 & 10,3 & 53,3 & 36,4 & 0,0 \\
4 & 9,2 & 52,9 & 37,9 & 0,0 & 10,3 & 51,5 & 36,2 & 2,0 \\
6 & 9,2 & 53,2 & 31,8 & 5,8 & 10,3 & 49,7 & 35,8 & 4,2 \\
8 & 9,2 & 53,2 & 29,6 & 8,0 & 10,3 & 52,1 & 31,3 & 6,3 \\
10 & 9,2 & 50,9 & 27,4 & 12,5 & 10,3 & 50,7 & 32,6 & 6,4 \\
12 & 9,2 & 56,4 & 21,9 & 12,5 & 10,3 & 52,5 & 30,0 & 7,2 \\
14 & 9,2 & 55,9 & 19,7 & 15,2 & 10,3 & 54,5 & 23,7 & 11,5 \\
16 & 9,2 & 56,0 & 17,1 & 17,7 & 10,3 & 53,5 & 22,2 & 14,0 \\
18 & 9,2 & 54,1 & 16,9 & 19,8 & 10,3 & 53,5 & 20,2 & 16,0 \\
20 & 9,2 & 54,3 & 16,4 & 20,1 & 10,3 & 52,7 & 17,3 & 19,7 \\
22 & 9,2 & 53,9 & 15,9 & 21,0 & 10,3 & 53,3 & 13,7 & 22,7 \\
24 & 9,2 & 52,0 & 17,9 & 20,9 & 10,3 & 51,7 & 14,4 & 23,6 \\
26 & 9,2 & 53,9 & 17,2 & 19,7 & 10,3 & 52,8 & 13,2 & 23,7 \\
\hline
\end{tabular}

ajusta con un error de 6,9\% los datos experimentales obtenidos de la probeta sin elementos aleantes y con un $21,7 \%$ los datos obtenidos de las probetas aleadas con cobre y níquel. En cuanto al parámetro $\alpha$, cuya magnitud varía inversamente con la energía de falla de apilamiento ${ }^{[21]}$, se observa que presenta una mayor magnitud para la muestra sin elementos aleantes. Lo anterior se entiende considerando que el cobre ${ }^{[25]}$ y níquel ${ }^{[26]}$ tienden a aumentar la energía de falla de apilamiento de la austenita, lo cual desfavorece la transformación martensítica, lo que podría explicar la elevada tenacidad y resistencia a la fatiga que presentan estas aleaciones. En relación al parámetro $\beta$, el cual se encuentra relacionado con la máxima cantidad de martensita transformada, se aprecia que este parámetro presenta un valor mayor en la muestra con elementos aleantes, donde el volumen de martensita trasformado alcanzó un $24 \%$. En relación al exponente n, se concluye que no se ve afectado por la composición de la muestra, manteniéndose en un valor de 4,4, muy cercano al valor reportado para aceros inoxidables $304(n=4,5)^{[21]}$.

La figura 9 muestra los ajustes obtenidos utilizando el modelo de Chang et al. ${ }^{[19]}$. Se determinó que el modelo ajusta con un error de 5,6 \% los datos experimentales obtenidos de la probeta sin elementos aleantes y con un $20,8 \%$ los datos obtenidos de las pro- betas aleadas con cobre y níquel. Respecto al parámetro $\beta$, el cual guarda relación inversa con la estabilidad termodinámica de la austenita, se observa que es mayor en la muestra sin elementos aleantes. Este resultado se explica considerando que tanto el cobre como el níquel son elementos estabilizadores de la austenita. El parámetro $\varepsilon_{\mathrm{o}}$, el cual representa la deformación mínima para comenzar la transformación martensítica, resultó idéntico para ambas muestras. Este resultado difiere de lo que experimentalmente se verificó, ya que para la muestra sin elementos aleantes sólo se constató la transformación martensítica sobre el $4 \%$ de deformación. Lo anterior pudo deberse a que el límite de resolución de la técnica de DRX se encuentra sobre el $2 \%$. En cuanto al índice $n$, el cual guarda relación con el modo de deformación, se observa que no es afectado en gran medida por la composición y presenta un valor menor al reportado para aceros inoxidables $304(n=2,2)$ sometidos a tracción.

Con el fin de establecer si los datos obtenidos con los modelos representan estadísticamente los datos experimentales, se realizó una prueba de hipótesis con un $\alpha=0,05$ (nivel de significancia del $5 \%$ ) utilizando una distribución compuesta por la diferencia entre los datos reales y modelados, donde la hipótesis nula fue $\mu_{0}=0$ (media es igual a cero), mientras que la hipótesis alternativa fue $\mu_{0} \neq 0$ 

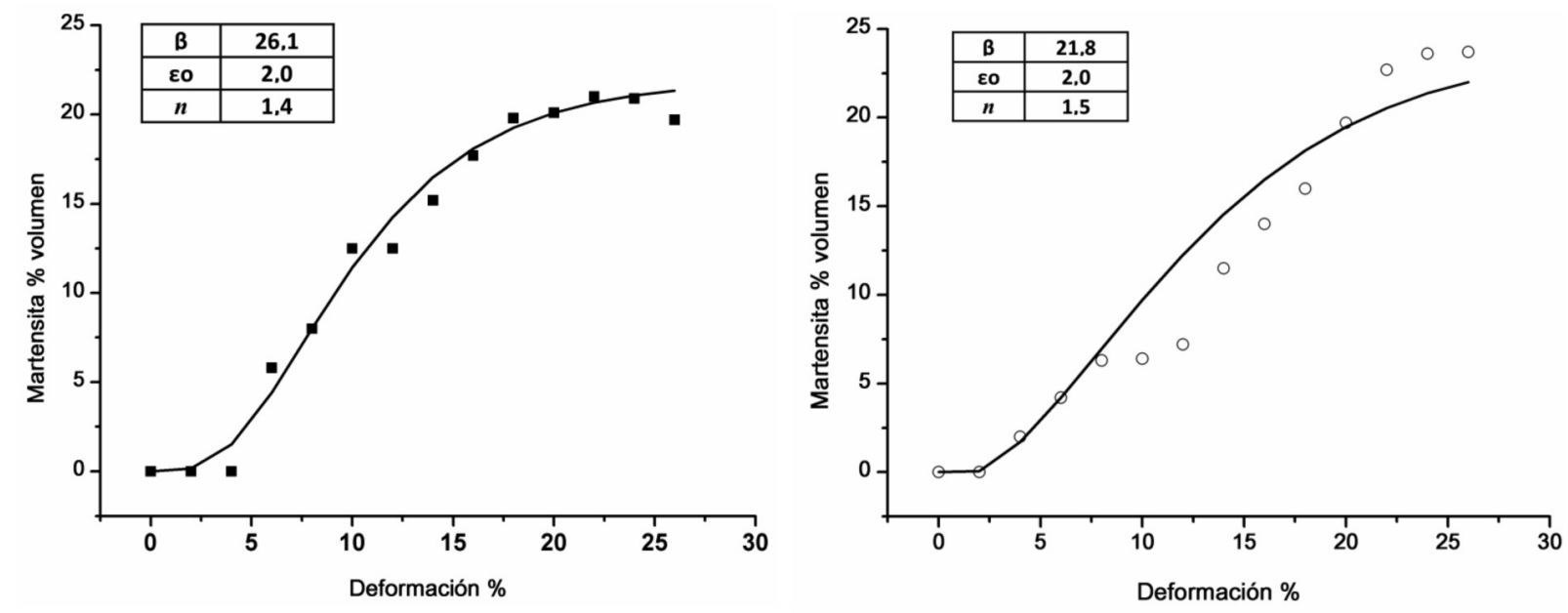

Figura 9. Comparación entre las curvas de transformación calculadas usando el modelo de Chang et al. y los datos experimentales (a) muestra sin aleantes y (b) muestra aleada con cobre y níquel.

Figure 9. Comparison between the calculated transformation curves obtained using the Chang et al. model and the experimental data (a) sample without alloying elements and (b) sample alloyed with copper and nickel.

(media distinta de cero). Los resultados obtenidos pueden ser apreciados en la tabla III, la cual presenta los intervalos de confianza para la media calculados con un nivel de confianza del $95 \%$. Basados en los resultados obtenidos, donde se aprecia que el cero se encuentra incluido en los intervalos de confianza calculados, se puede concluir que estadísticamente tanto el modelo de Olson-Cohen y Chang et al., representan la transformación martensítica inducida por deformación en fundiciones nodulares austemperadas.

Como se aprecia en la tabla III, la amplitud de los intervalos es mayor en los resultados obtenidos a partir de las fundiciones con elementos aleantes en comparación con los obtenidos de las fundiciones sin elementos aleantes, lo cual concuerda con el mayor error entre los datos predichos por ambos modelos y los datos obtenidos experimentalmente para este tipo de aleaciones (error $\approx 21 \%$ ). Basado en lo anterior, se establece que aun cuando tanto los modelos de Olson-Cohen ${ }^{[21]}$ y Chang et. al ${ }^{[19]}$ representan estadísticamente la transformación martensítica en fundiciones nodulares austemperadas con presencia de cobre y níquel, se debe profundizar en el estudio del efecto de estos elementos aleantes sobre la fenomenología de la transformación martensítica inducida por deformación en estas aleaciones.

Tabla III. Intervalos de confianza del $95 \%$ para la media

Table III. $95 \%$ confidence interval for the mean

\begin{tabular}{llc}
\hline Muestras & Modelos & $\begin{array}{c}\text { Intervalo de confianza } \\
\text { del } 95 \% \text { para la medida }\end{array}$ \\
\hline Sin aleantes & Olson-Cohen & {$[-0,0077: 0,0047]$} \\
& Chang et al. & {$[-0,0078: 0,0048]$} \\
Con aleantes & Olson-Cohen & {$[-0,0086: 0,0112]$} \\
& Chang et al. & {$[-0,0224: 0,0047]$} \\
\hline
\end{tabular}




\section{CONCLUSIONES}

Basados en los resultados obtenidos en el presente trabajo se concluye que:

- Se verificó la transformación martensítica inducida por deformación en ambas muestras bajo estudio.

- Tanto los modelos propuestos por OlsonCohen ${ }^{[21]}$ y Chang et al. ${ }^{[19]}$ para aceros inoxidables austeníticos pueden representar la cinética de transformación martensítica inducida por deformación en fundiciones nodulares austemperadas sin aleantes (con error $<7 \%$ ). Para las fundiciones aleadas con cobre y níquel los datos se ajustaron con un nivel de error $<22 \%$.

- El efecto del cobre y níquel dificulta la transformación martensítica inducida por deformación debido a que estos elementos aumentan la energía de falla de apilamiento, disminuyendo la zona fallada, lo cual se ve reflejado en el valor del parámetro $\alpha$ del modelo de Olson-Cohen ${ }^{[21]}$, además de aumentar la estabilidad de la austenita, lo que se verifica en la magnitud del parámetro $\beta$ del modelo Chang et al. ${ }^{[19]}$.

- La presencia de cobre y níquel, al retardar la transformación martensítica inducida por deformación, influiría en la elevada tenacidad y resistencia a la fatiga que presentan estas aleaciones, y por el contrario, tendría un efecto negativo sobre la resistencia al desgaste.

\section{Agradecimientos}

Los autores agradecen a los proyectos FONDECYT $\mathrm{N}^{\circ} 1990866$ de CONICYT y al proyecto DIUDA 221159 de la Universidad de Atacama, por el financiamiento de este trabajo. Además se agradece a los Profesores M. Guevara, J. Astorga y E. Cárdenas, por el apoyo prestado.

\section{REFERENCIAS}

[1] Y. Kim, H. Shin, H. Park y J. Lim, Mater. Lett. 62 (2008) 357-360.

[2] J. Aranzábal, I. Gutiérrez, J.M. Rodriguez y J.J. Urcola, J. Mater. Sci. Technol. 8 (1992) 263 273.

[3] T. Tun y K. Lwin, Journal of Metals, Materials and Minerals 18 (2008) 199-205.

[4] J.L. Garin y R.L. Mannheim, J. Mater. Process. Technol. 143-144 (2003) 347-351.
[5] D.J. Moore, T.N. Rouns y K.B. Rundamm, J. Heat Treat. 4 (1985) 7-24.

[6] S. Daber y P. Rao, J. Mater. Sci. 43 (2008) 357 367.

[7] J.R. Davis, ASM Specialty Handbook: Cast Irons, ASM International, EE.UU., 1996, pp. 192-204.

[8] T. Rouns y K. Rundman, AFS Transactions 95 (1987) 851-874.

[9] D. Rousière y J. Aranzabal, Metallurgical Science and Technology 18 (2000) 24-29.

[10] B. Kovacs, World conference on Austempered Ductile Iron, Chicago, EE.UU., 1991, pp. 241270.

[11] C. Hsu y K. Lin, Mat. Sci. Eng. A 528 (2011) 5.706-5.712.

[12] O. Erić, D. Rajnović, S. Zec, L. Sidjanin y M. Jovanović, Mater. Charact. 57 (2006) 211- 217.

[13] O. Erić, D. Rajnović, L. Sidjanin, S. Zec y M. Jovanović, J. Serb. Chem. Soc. 70 (2005) 1.0151.022 .

[14] J. Yang y S. Putatunda, Mat. Sci. Eng. A 406 (2005) 217-228.

[15] P. Shanmugam, P. Prasad, K. Udupa y N. Venkataraman, J. Mater. Sci. 29 (1994) 4.933. 4.940 .

[16] M. Bahmani y R. Elliott, J. Mater. Sci. 32 (1997) 5.383-5.388.

[17] Qit-Fer et Titane Inc, Ductile iron data for design engineers, Rio Tinto \& Titanium Inc., Canada, 1990, p. 26.

[18] J. Zimba, D.J. Simbi y E. Navara, Cement Concrete Comp. 25 (2003) 643-649.

[19] H. Shih, T. Ha y Y. Chang, Scripta Mater. 45 (2001) 823-829.

[20] S. Chatterjee y H. Bhadeshia, Mater. Sci. Tech. 22 (2006) 645-649.

[21] G. Olson y M. Cohen, Metall. Mater.Trans. A, 6 (1975) 791-795.

[22] ASM Handbook, Metallography and Microstructures, Vol 9. ASM International, EE. UU., 1992.

[23] G.I. Sil'man, V.V. Kamynin y A.A. Tarasov, Met. Sci. Heat Treat. 45 (2003) 254-258.

[24] R.A. Gonzaga, P. Martínez, A. Pérez y P. Villanueva, Journal of Achievements in Materials and Manufacturing Engineering 33 (2009) 150-158.

[25] A. Dumay, J.P. Chateau, S. Allain, S. Migot y O. Bouaziz, Mat. Sci. Eng. A 483-484 (2008) 184-187.

[26] J. Liu, P. Han, M. Dong, G. Fan, G. Qiao y J. Yang, Physica B 407(2012) 891-895. 